\title{
Elucidating the Short Term Loss Behavior of Markovian-Modulated Batch-Service Queueing Model with Discrete-Time Batch Markovian Arrival Process
}

\author{
Yung-Chung Wang, Dong-Liang Cai, Li-Hsin Chiang, and Cheng-Wei Hu
}

Department of Electrical Engineering, National Taipei University of Technology, Taipei 10608, Taiwan

Correspondence should be addressed to Yung-Chung Wang; ycwang@ntut.edu.tw

Received 6 October 2013; Revised 23 December 2013; Accepted 23 December 2013; Published 26 March 2014

Academic Editor: Cristian Toma

Copyright (C) 2014 Yung-Chung Wang et al. This is an open access article distributed under the Creative Commons Attribution License, which permits unrestricted use, distribution, and reproduction in any medium, provided the original work is properly cited.

\begin{abstract}
This paper applies a matrix-analytical approach to analyze the temporal behavior of Markovian-modulated batch-service queue with discrete-time batch Markovian arrival process (DBMAP). The service process is correlated and its structure is presented through discrete-time batch Markovian service process (DBMSP). We examine the temporal behavior of packet loss by means of conditional statistics with respect to congested and noncongested periods that occur in an alternating manner. The congested period corresponds to having more than a certain number of packets in the buffer; noncongested period corresponds to the opposite. All of the four related performance measures are derived, including probability distributions of a congested and noncongested periods, the probability that the system stays in a congested period, the packet loss probability during congested period, and the long term packet loss probability. Queueing systems of this type arise in the domain of wireless communications.
\end{abstract}

\section{Introduction}

The queueing systems under various types of arrival and service processes have been investigated due to their applicability in wireless multimedia networks. The bursty nature of multimedia traffic incurs bursty packet losses, making it impossible for long term loss behavior to quantify precisely the QoS of multimedia services over the internet. However, the quality of real-time multimedia applications perceived by the end user is highly sensitive to the bursty loss behavior, necessitating the supplementing of traditional long term QoS metrics with short term based ones. Therefore, short term performance metrics such as distribution of the length of congested periods and noncongested periods, that is, the likelihood that the system remains in the congested period and loss probability during the congested period, will seriously degrade the quality of service perceived by the end users, which is especially relevant for systems supporting realtime multimedia services. Consequently, short term performance metrics profoundly impact the evaluation of wireless based multimedia applications. Exploring the short term loss behavior of multimedia traffic in wireless multimedia networks is thus of priority concern.

Because the traditional Markov model can not adequately capture the property of complex multimedia traffic, such as web browsing, VoIP, and teleconferencing, it is necessary to propose a suitable traffic model to describe multimedia applications over wireless networks. Traffic with certain bursty characteristics can be qualitatively modeled by a DBMAP, as confirmed in [1]. The literature on the application of the DBMAP to model network traffic is rich. The use of DBMAP is proposed in [2] to model video sources. In [3], Kang et al. provided evidence that DMAP yields a very good estimation of the cell loss ratio for usual superpositions of voice and VBR video sources. In [4], Heyman and Lucantoni provided evidence that a DMMPP is a good model for Internet traffic at the packet/byte level. In [5], Salvador et al. proposed a parameter fitting procedure using superposed two-state DMMPP that leads to accurate estimates of queueing behavior for network traffic exhibiting long-range-dependent behavior. 
Based on this observation, an arrival process for packet stream is modeled as a DBMAP $[1,2,6]$ in this paper.

Like the DBMAP, discrete-time batch Markovian service process (DBMSP) is a versatile service process and can capture the correlation among the service times. Therefore, DBMSP is suitable to model wireless systems employing multiple transmission modes in the physical layer, each of which corresponds to a particular modulation and coding scheme. Based on this observation, service process for wireless multimedia networks is modeled as a DBMSP in this paper.

So far, much research has focused on the queue with Markovian service process (MSP). In [7], Bocharov et al. applied the method of embedded Markov chain to derive the main stationary characteristics of the G/MSP/1/r. In [8], Banik and Gupta derived the steady state distribution at prearrival and arbitrary epochs along with some important performance measures of $\mathrm{GI}^{X} / \mathrm{MSP} / 1 / \mathrm{N}$. In [9], Gupta and Banik derived system length distribution at prearrival and arbitrary epochs for finite and infinite buffer single server queue with renewal input and Markovian service process. In [10], Wang et al. derived the loss pattern of DBMAP/DMSP/1/K. In [11], Samanta and Zhang analyzed the steady state behavior of a discrete-time single-server queueing system with MSP and server vacations. However, all of the above papers consider the queue with MSP. These models can not be applied to analyze the performance measures of wireless multimedia networks. So far, much research has focused on the batch-service queue. In [12], Chaudhry and Gupta analyzed a finite buffer discrete-time queue with general bulk-service rule, wherein the arrival process is DMAP and service times are arbitrarily and independently distributed. In [13], Banik obtained queue length distributions at various epochs of $\mathrm{BMAP} \mathrm{G}^{(a, b)} / 1 / \mathrm{N}$ and BMAP/MSP ${ }^{(a, b)} / 1 / \mathrm{N}$ systems. In [14], Claeys et al. derived moments and tail probabilities of the customer delay in a discrete-time queueing system with batch arrivals and a batch server adopting the full batch-service policy. In [15], Claeys et al. studied the the customer delay and buffer content of the discrete-time $\mathrm{Geo}^{X} / \mathrm{G}^{(l, c)} / 1$ system. In [16], Claeys et al. analyzed a discrete-time DBMAP/G $\mathrm{G}^{(l, c)} / 1$ queue, whereby the service time of a batch is dependent on the number of customers within it. Most of the past works assume an infinite buffer and focus on obtaining the queue and the waiting time characteristics. This paper assumes a finite buffer and obtains various packet loss characteristics such as the congested period, noncongested period, and the packet loss probability during congested period. With buffer size $K$, the sojourntime on congestion of the DBMAP/DBMSP/1/K queueing system is characterized. Mathematical models undertaken here to examine the short term packet loss behavior of a queue are different from those in the literature in one or more respects. We apply a matrix-analytical approach to analyze the sojourn-time on congestion. We examine the bursty nature of packet loss by means of conditional statistics with respect to congested and noncongested periods that occur in an alternating manner. As stated before, the congested period corresponds to having more than a certain number of packets in the buffer; the noncongested period corresponds to the opposite. Hence, there is a threshold buffer level that splits the state space in two. By such state-space decomposition, two transient Markov chains are devised to describe the alternating renewal process. The distributions of various absorbing times in the two transient Markov chains are derived to compute the average durations of the congested periods and the conditional packet loss probability encountered during a congested period. These performance measures describe the quality of service provided by the communication devices with wireless link.

This paper is organized as follows. In Section 2, the DBMAP/DBMSP/1/K queueing system is briefly introduced. In Section 3, the short term loss behavior of the DBMAP/DBMSP/ $1 / K$ queueing system is analyzed. In Section 4, Queueing systems of this type occur in the domain of wireless communications. Experimental numerical results are computed and discussed in Section 5 to reveal the computational tractability of our analysis and to develop a better understanding of the packet loss pattern over wireless networks. Concluding remarks are given in Section 6.

\section{DBMAP/DBMSP/ $1 / K$ Queue}

In this section, we introduce the arrival process, service process, and queueing model.

2.1. Arrival Model. The arrival processes discussed in this paper are assumed to be DBMAPs since time is assumed to be slotted. A DBMAP can be described by a special type of discrete-time Markov chain. Let $\left\{\left(N_{a}(n), J_{a}(n)\right)\right\}_{n \geq 0}$ be a discrete-time Markov chain with two-dimensional state space $\left\{(l, j) \mid l \geq 0,1 \leq j \leq m_{a}\right\}$ and transition probability matrix

$$
\left[\begin{array}{ccccc}
\mathrm{D}_{0} & \mathrm{D}_{1} & \mathrm{D}_{2} & \mathrm{D}_{3} & \cdots \\
\mathbf{0} & \mathrm{D}_{0} & \mathrm{D}_{1} & \mathrm{D}_{2} & \cdots \\
\mathrm{0} & \mathbf{0} & \mathrm{D}_{0} & \mathrm{D}_{1} & \cdots \\
& & & \cdot & \cdots
\end{array}\right]
$$

where $N_{a}(n)$ represents the total number of arrivals during $n$ consecutive slots, $J_{a}(n)$ represents an auxiliary state or phase variable, and $\mathbf{D}_{\mathbf{i}}$ s are nonnegative $m_{a} \times m_{a}$ matrices whose entries are between 0 and 1 , called parameter matrices. The transition probability from state $(l, j)$ to state $\left(l+i, j^{\prime}\right)$, which corresponds to the arrival of a batch of size $i$, is the $\left(j, j^{\prime}\right)$ th entry $\left(\mathbf{D}_{\mathbf{i}}\right)_{j, j^{\prime}}$ of the $m_{a} \times m_{a}$ matrix $\mathbf{D}_{\mathbf{i}} \cdot\left(\mathbf{D}_{\mathbf{i}}\right)_{j, j^{\prime}}$ may depend on phases $j$ and $j^{\prime}$. The sum of all parameter matrices

$$
\mathbf{D}=\sum_{i=0}^{\infty} \mathbf{D}_{\mathbf{i}}
$$

is an $m_{a} \times m_{a}$ stochastic matrix which is the transition probability matrix of the underlying Markovian structure $\left\{J_{a}(n)\right\}_{n \geq 0}$ with respect to the DBMAP. $\left(\mathbf{I}-\mathbf{D}_{\mathbf{0}}\right)$ is assumed to be nonsingular such that the sojourn time at any state of the state space $\left\{(l, j) \mid l \geq 0,1 \leq j \leq m_{a}\right\}$ is finite with probability 1 , thus guaranteeing that the process never 
terminates. The fundamental arrival rate $\lambda$ of this DBMAP can then be defined as

$$
\lambda=\pi_{\mathbf{a}}\left(\sum_{i=1}^{\infty} i \mathbf{D}_{\mathbf{i}}\right) \mathbf{e},
$$

where $\pi_{\mathbf{a}}$ is the stationary probability vector of $\mathbf{D}$ in (2); that is, $\boldsymbol{\pi}_{\mathbf{a}} \mathbf{D}=\pi_{\mathrm{a}}, \boldsymbol{\pi}_{\mathrm{a}} \mathbf{e}=1$, and $\mathbf{e}$ is assumed in this paper to be the all-1 column vector with the designated dimension.

2.2. Service Model. A DBMSP can be described by a special type of discrete-time Markov chain. Let $\left\{\left(N_{s}(n), J_{s}(n)\right)\right\}_{n \geq 0}$ be a discrete-time Markov chain with two-dimensional state space $\left\{(l, j) \mid l \geq 0,1 \leq j \leq m_{s}\right\}$ and transition probability matrix

$$
\left[\begin{array}{ccccc}
\mathbf{H}_{0} & \mathrm{H}_{1} & \mathbf{H}_{2} & \mathbf{H}_{3} & \cdots \\
\mathbf{0} & \mathrm{H}_{0} & \mathrm{H}_{1} & \mathbf{H}_{2} & \cdots \\
\mathbf{0} & \mathbf{0} & \mathrm{H}_{0} & \mathbf{H}_{1} & \cdots \\
& & & \cdot & \cdots
\end{array}\right]
$$

where $N_{s}(n)$ represents the total number of served packets during $n$ consecutive slots, $J_{s}(n)$ represents an auxiliary state or phase variable, and $\mathbf{H}_{\mathbf{i}}, \quad 0 \leq i \leq M_{s}$, are nonnegative $m_{s} \times m_{s}$ matrices whose entries are between 0 and 1 , called parameter matrices. $M_{s}$ is the maximum number of packets to be served at one slot time. The transition probability from state $(l, j)$ to state $\left(l+i, j^{\prime}\right)$, which corresponds to the service departure of a batch of size $i$, is the $\left(j, j^{\prime}\right)$ th entry $\left(\mathbf{H}_{\mathbf{i}}\right)_{j, j^{\prime}}$ of the $m_{s} \times m_{s}$ matrix $\mathbf{H}_{\mathbf{i}} \cdot\left(\mathbf{H}_{\mathbf{i}}\right)_{j, j^{\prime}}$ may depend on phases $j$ and $j^{\prime}$. The sum of all parameter matrices

$$
\mathbf{H}=\sum_{i=0}^{M_{s}} \mathbf{H}_{\mathbf{i}}
$$

is an $m_{s} \times m_{s}$ stochastic matrix which is the transition probability matrix of the underlying Markovian structure $\left\{J_{s}(n)\right\}_{n \geq 0}$ with respect to the DBMSP. $\left(\mathbf{I}-\mathbf{H}_{\mathbf{0}}\right)$ is assumed to be nonsingular such that the sojourn time at any state of the state space $\left\{(l, j) \mid l \geq 0,1 \leq j \leq m_{s}\right\}$ is finite with probability 1 , thus guaranteeing that the process never terminates. The fundamental service rate $\mu$ of this DBMSP can then be defined as

$$
\mu=\pi_{\mathbf{s}}\left(\sum_{i=1}^{M_{s}} i \mathbf{H}_{\mathbf{i}}\right) \mathbf{e}
$$

where $\boldsymbol{\pi}_{\mathbf{s}}$ is the stationary probability vector of $\mathbf{H}$ in (5); that is, $\boldsymbol{\pi}_{\mathbf{s}} \mathbf{H}=\boldsymbol{\pi}_{\mathbf{s}}, \boldsymbol{\pi}_{\mathbf{s}} \mathbf{e}=1$, and $\mathbf{e}$ is assumed in this paper to be the all-1 column vector with the designated dimension.

2.3. DBMAP/DBMSP/1/K Queue. The queueing model must be specified as the late arrival model, in which a packet arrives to the queue before a slot boundary. Consider the embedded Markov chain $\{(L(n), J(n), R(n))\}_{n \geq 0}$ of the queueing system, which can be described as a particular type of semi-Markov process where the state jumps regularly at a constant slot time. This is considered in the state space $\Omega=\{0,1, \ldots, K\} \times$ $\left\{1,2, \ldots, m_{a}\right\} \times\left\{1,2, \ldots, m_{s}\right\}$, where $L(n), J(n)$, and $R(n)$ denote the buffer occupancy, the phase of the DBMAP, and the phase of the DBMSP, respectively, at the end of the $n$th time slot. For convenience, the queueing system is said to be at a level $j$ if its buffer occupancy is equal to $j$. The Markov chain now has an irreducible transition probability matrix of the following block form:

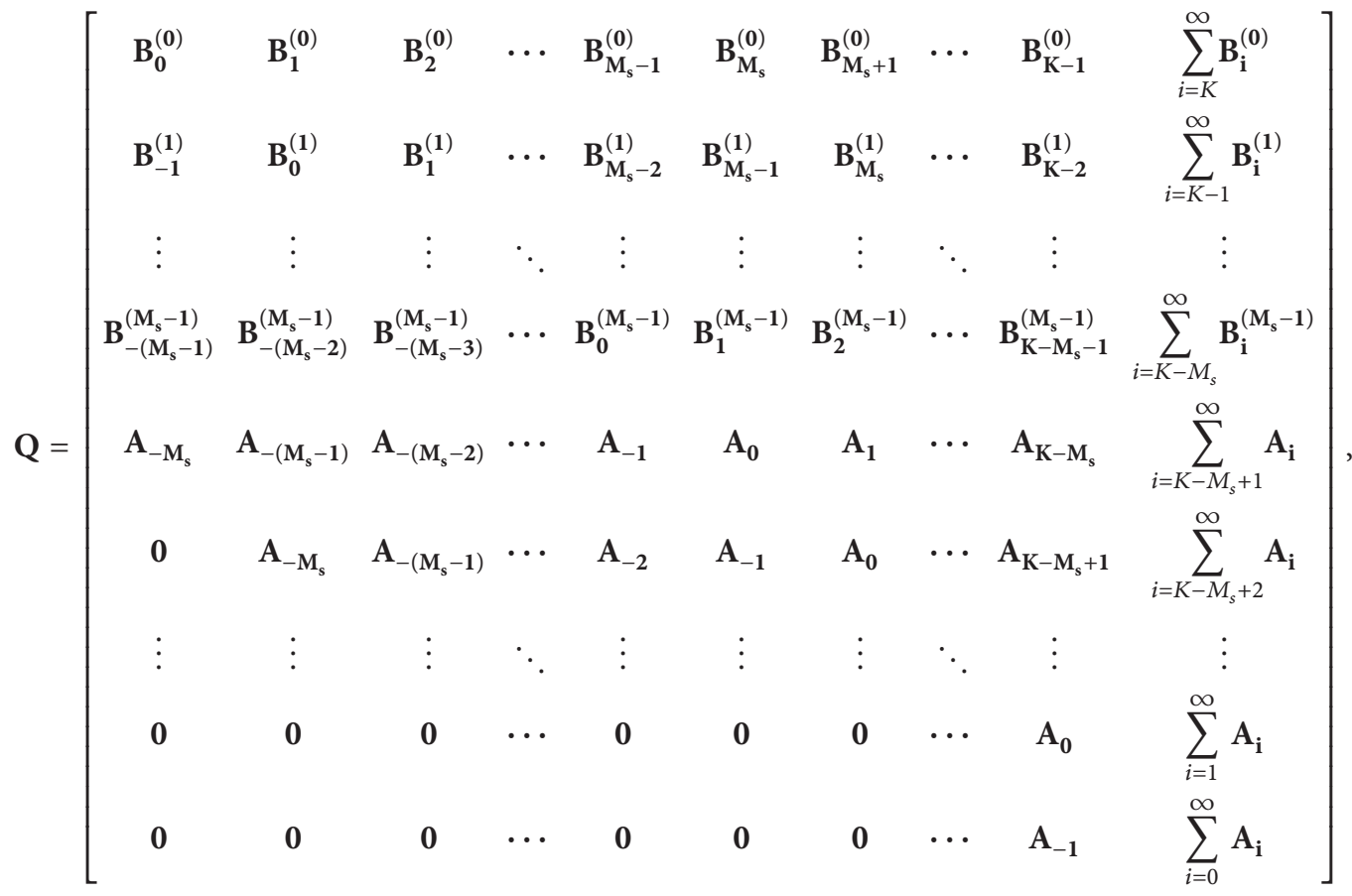


where

$$
\begin{gathered}
\mathbf{B}_{\mathbf{i}}^{(\mathbf{0})}=\mathbf{D}_{\mathbf{i}} \otimes \mathbf{H}, \quad 0 \leq i \leq \infty, \\
\mathbf{B}_{-\mathbf{i}}^{(\mathbf{i})}=\mathbf{D}_{\mathbf{0}} \otimes \sum_{k=i}^{M_{s}} \mathbf{H}_{\mathbf{k}}, \quad 1 \leq i \leq M_{s}-1, \\
\mathbf{B}_{-\mathbf{i}}^{(\mathbf{j})}=\sum_{k=0}^{j-i-1} \mathbf{D}_{\mathbf{k}} \otimes \mathbf{H}_{\mathbf{i}+\mathbf{k}}+\mathbf{D}_{\mathbf{j}-\mathbf{i}} \otimes \sum_{k=j}^{M_{s}} \mathbf{H}_{\mathbf{k}}, \\
1 \leq i \leq j, \quad 2 \leq j \leq M_{s}-1, \\
\mathbf{B}_{\mathbf{i}}^{(\mathbf{j})}=\sum_{k=0}^{j-1} \mathbf{D}_{\mathbf{i}} \otimes \mathbf{H}_{\mathbf{k}}+\mathbf{D}_{\mathbf{i}+\mathbf{j}} \otimes \sum_{k=j}^{M_{s}} \mathbf{H}_{\mathbf{k}}, \\
0 \leq i \leq \infty, \quad 1 \leq j \leq M_{s}-1, \\
\mathbf{A}_{-\mathbf{i}}=\sum_{k=i}^{M_{s}} \mathbf{D}_{\mathbf{k}-\mathbf{i}} \otimes \mathbf{H}_{\mathbf{k}}, \quad 0<i \leq M_{s}, \\
\mathbf{A}_{\mathbf{i}}=\sum_{k=0}^{M_{s}} \mathbf{D}_{\mathbf{k}+\mathbf{i}} \otimes \mathbf{H}_{\mathbf{k}}, \quad 0 \leq i \leq \infty
\end{gathered}
$$

and $\otimes$ is the Kronecker product [17]. Each block is of dimension $m_{a} m_{s} \times m_{a} m_{s}$ and corresponds to the transition from one buffer level to another buffer level.

\section{Performance Evaluation}

To characterize the real packet loss behavior of wireless communication, it is not adequate to examine only the long term packet loss probabilities. For example, a packet stream may experience the loss of a string of consecutive packets followed by bursty arrivals, though the long term packet loss probability is small. This phenomenon makes the traffic source suffer from a significant QoS degradation in that time period. Therefore, in light of the high correlation among consecutive packet arrivals in the wireless multimedia networks, it is necessary to study the packet loss behavior during a short term interval, that is, the conditional packet loss behavior, as well as during long term intervals, in order to characterize the real packet loss behavior of a wireless communication queueing system.

3.1. Transient Performance Measures. The level of buffer occupancy of a queueing system passes through alternating congested and noncongested periods. To study the short term loss behavior of a queue during a congested period, we decompose the state space $\Omega$ into two subsets $\Omega_{n c} \cup \Omega_{c}$ according to the congested buffer level $k_{\text {th }}$. In order to simplify the description, we assume $k_{\text {th }}>M_{s}$ in this paper. With this partition of the state space, the transition probability matrix $\mathbf{Q}$ in (7) of the Markov chain of the queueing system can be partitioned as follows:

$$
\mathbf{Q}=\left[\begin{array}{cc}
\mathbf{Q}_{\mathrm{nc}} & \mathbf{Q}_{\mathrm{nc}, \mathrm{c}} \\
\mathbf{Q}_{\mathrm{c}, \mathrm{nc}} & \mathbf{Q}_{\mathrm{c}}
\end{array}\right],
$$

where

$$
\begin{aligned}
& \mathbf{Q}_{\mathbf{n c}}=\left[\begin{array}{ccccc}
\mathbf{B}_{0}^{(0)} & \mathbf{B}_{1}^{(0)} & \cdots & \mathbf{B}_{\mathrm{k}_{\mathrm{h}}-2}^{(0)} & \mathbf{B}_{\mathrm{k}_{\mathrm{th}}-1}^{(0)} \\
\mathbf{B}_{-1}^{(1)} & \mathbf{B}_{0}^{(1)} & \cdots & \mathbf{B}_{\mathrm{k}_{\mathrm{th}}-3}^{(1)} & \mathbf{B}_{\mathbf{k}_{\mathrm{h}}-2}^{(1)} \\
\mathbf{B}_{-2}^{(2)} & \mathbf{B}_{-1}^{(2)} & \cdots & \mathbf{B}_{\mathrm{k}_{\mathrm{th}}-4}^{(2)} & \mathbf{B}_{\mathbf{k}_{\mathrm{th}}-3}^{(2)} \\
\vdots & \vdots & \ddots & \vdots & \vdots \\
\mathbf{0} & \mathbf{0} & \cdots & \mathbf{A}_{-1} & \mathbf{A}_{\mathbf{0}}
\end{array}\right], \\
& \mathbf{Q}_{\mathbf{n c}, \mathbf{c}}=\left[\begin{array}{ccccc}
\mathbf{B}_{\mathbf{k}_{\mathrm{th}}}^{(\mathbf{0}} & \mathbf{B}_{\mathbf{k}_{\mathrm{th}}+\mathbf{1}}^{(\mathbf{0})} & \cdots & \mathbf{B}_{\mathbf{K}-\mathbf{1}}^{(\mathbf{0})} & \sum_{i=K}^{\infty} \mathbf{B}_{\mathbf{i}}^{(\mathbf{0})} \\
\mathbf{B}_{\mathbf{k}_{\mathrm{th}}-\mathbf{1}}^{(1)} & \mathbf{B}_{\mathbf{k}_{\mathrm{th}}}^{(\mathbf{1})} & \cdots & \mathbf{B}_{\mathrm{K}-2}^{(1)} & \sum_{i=K-1}^{\infty} \mathbf{B}_{\mathbf{i}}^{(1)} \\
\mathbf{B}_{\mathbf{k}_{\mathrm{th}}-2}^{(2)} & \mathbf{B}_{\mathbf{k}_{\mathrm{th}}-\mathbf{1}}^{(2)} & \cdots & \mathbf{B}_{\mathrm{K}-3}^{(2)} & \sum_{i=K-2}^{\infty} \mathbf{B}_{\mathbf{i}}^{(2)} \\
\vdots & \vdots & \ddots & \vdots & \vdots \\
\mathbf{A}_{1} & \mathbf{A}_{2} & \cdots & \mathbf{A}_{\mathbf{K}-\mathbf{k}_{\mathrm{th}}-4} & \sum_{i=K-k_{t h}-3}^{\infty} \mathbf{A}_{\mathbf{i}}
\end{array}\right], \\
& \mathbf{Q}_{\mathbf{c}, \mathbf{n c}}=\left[\begin{array}{ccccccc}
\mathbf{0} & \cdots & \mathbf{0} & \mathbf{A}_{-\mathrm{M}_{s}} & \cdots & \cdots & \mathbf{A}_{-\mathbf{1}} \\
\mathbf{0} & \cdots & \mathbf{0} & \mathbf{0} & \mathbf{A}_{-\mathbf{M}_{s}} & \cdots & \mathbf{A}_{-2} \\
\vdots & \ddots & \vdots & \vdots & \vdots & \ddots & \vdots \\
\mathbf{0} & \cdots & \mathbf{0} & \mathbf{0} & \mathbf{0} & \cdots & \mathbf{A}_{-\mathbf{M}_{s}} \\
\vdots & \ddots & \vdots & \vdots & \vdots & \ddots & \vdots \\
\mathbf{0} & \cdots & \mathbf{0} & \mathbf{0} & \mathbf{0} & \cdots & \mathbf{0}
\end{array}\right] \text {, }
\end{aligned}
$$

$\mathbf{Q}_{\mathrm{c}}$

$$
=\left[\begin{array}{cccccc}
\mathbf{A}_{\mathbf{1}} & \mathbf{A}_{\mathbf{2}} & \cdots & \mathbf{A}_{\mathbf{M}_{\mathrm{s}}} & \cdots & \sum_{i=K-k_{\mathrm{th}}-1}^{\infty} \mathbf{A}_{\mathbf{i}} \\
\mathbf{A}_{\mathbf{0}} & \mathbf{A}_{\mathbf{1}} & \cdots & \mathbf{A}_{\mathbf{M}_{\mathrm{s}}-\mathbf{1}} & \cdots & \sum_{i=K-k_{\mathrm{th}}}^{\infty} \mathbf{A}_{\mathbf{i}} \\
\vdots & \vdots & \ddots & \vdots & \ddots & \vdots \\
\mathbf{A}_{-\mathbf{M}_{\mathrm{s}}} & \mathbf{A}_{-\left(\mathbf{M}_{\mathrm{s}}-\mathbf{1}\right)} & \cdots & \mathbf{A}_{\mathbf{0}} & \cdots & \sum_{i=K-k_{\mathrm{th}}-M_{s}-1}^{\infty} \mathbf{A}_{\mathbf{i}} \\
\mathbf{0} & \mathbf{A}_{-\mathbf{M}_{\mathbf{s}}} & \cdots & \mathbf{A}_{-\mathbf{1}} & \cdots & \sum_{i=K-k_{\mathrm{th}}-M_{s}-2}^{\mathbf{A}_{\mathbf{i}}} \\
\vdots & \vdots & \ddots & \vdots & \ddots & \vdots \\
\mathbf{0} & \mathbf{0} & \cdots & \mathbf{A}_{-\mathbf{M}_{\mathbf{s}}} & \cdots & \sum_{i=0}^{\infty} \mathbf{A}_{\mathbf{i}}
\end{array}\right] .
$$

The matrices $\mathbf{Q}_{\mathbf{n c}}, \mathbf{Q}_{\mathbf{n c}, \mathbf{c}}, \mathbf{Q}_{\mathbf{c}, \mathbf{n c}}$, and $\mathbf{Q}_{\mathrm{c}}$ are transition probability submatrices governing transitions from $\Omega_{n c}$ into itself, from $\Omega_{n c}$ into $\Omega_{c}$, from $\Omega_{c}$ into $\Omega_{n c}$, and from $\Omega_{c}$ into itself, respectively.

Next, noncongested and congested periods are characterized by deriving the steady state probabilities for the initial 
state of each transient Markov chain, as denoted by vector $\beta_{n c}$ for noncongested and vector $\beta_{c}$ for congested periods. Then,

$$
\begin{array}{r}
\beta_{\mathrm{nc}}=\boldsymbol{\beta}_{\mathrm{nc}}\left(\left(\mathrm{I}-\mathbf{Q}_{\mathrm{nc}}\right)^{-1} \mathbf{Q}_{\mathrm{nc}, \mathrm{c}}\left(\mathrm{I}-\mathbf{Q}_{\mathrm{c}}\right)^{-1} \mathbf{Q}_{\mathrm{c}, \mathrm{nc}}\right), \\
\boldsymbol{\beta}_{\mathrm{nc}} \mathrm{e}=1, \\
\boldsymbol{\beta}_{\mathrm{c}}=\boldsymbol{\beta}_{\mathrm{c}}\left(\left(\mathrm{I}-\mathbf{Q}_{\mathrm{c}}\right)^{-1} \mathbf{Q}_{\mathrm{c}, \mathrm{nc}}\left(\mathrm{I}-\mathbf{Q}_{\mathrm{nc}}\right)^{-1} \mathbf{Q}_{\mathrm{nc}, \mathrm{c}}\right), \\
\boldsymbol{\beta}_{\mathrm{c}} \mathrm{e}=1 .
\end{array}
$$

Let $L_{n c}$ and $L_{c}$ be the lengths of noncongested and congested periods in the queuing system, respectively. Obviously, $L_{n c}$ and $L_{c}$ represent the life-span of the transient Markov chain, with respect to $Q_{n c}$ and $Q_{c}$ for transitions on $\Omega_{n c}$ and $\Omega_{c}$. According to the transient Markov chain theory, the respective probability generating functions of $P\left\{L_{n c}=k\right\}$ and $P\left\{L_{c}=k\right\}$ are

$$
\begin{gathered}
\widehat{P}_{L_{n c}}(z)=z \boldsymbol{\beta}_{\mathbf{n c}}\left(\mathbf{I}-z \mathbf{Q}_{\mathbf{n c}}\right)^{-1} \mathbf{Q}_{\mathbf{n c}, \mathbf{c}} \mathbf{e}, \\
\widehat{P}_{L_{c}}(z)=z \boldsymbol{\beta}_{\mathbf{c}}\left(\mathbf{I}-z \mathbf{Q}_{\mathbf{c}}\right)^{-1} \mathbf{Q}_{\mathbf{c}, \mathbf{n c}} \mathbf{e} .
\end{gathered}
$$

The average lengths of noncongested and congested periods are

$$
\begin{array}{r}
E\left[L_{n c}\right]=\left.\frac{d}{d z} \widehat{P}_{L_{n c}}(z)\right|_{z=1}=\boldsymbol{\beta}_{\mathbf{n c}}\left(\mathbf{I}-\mathbf{Q}_{\mathbf{n c}}\right)^{-2} \mathbf{Q}_{\mathbf{n c}, \mathbf{c}} \mathbf{e}, \\
E\left[L_{c}\right]=\left.\frac{d}{d z} \widehat{P}_{L_{c}}(z)\right|_{z=1}=\boldsymbol{\beta}_{\mathbf{c}}\left(\mathbf{I}-\mathbf{Q}_{\mathbf{c}}\right)^{-2} \mathbf{Q}_{\mathbf{c}, \mathbf{n c}} \mathbf{e} .
\end{array}
$$

To investigate the packet loss behavior during a congested period, the submatrix $\mathbf{Q}_{\mathbf{c}}$ in (9) is decomposed as

$$
\mathbf{Q}_{\mathbf{c}}=\mathbf{Q}_{\mathbf{c}}(0)+\sum_{\ell=1}^{\infty} \mathbf{Q}_{\mathbf{c}}(\ell)
$$

where

$$
\begin{aligned}
& \mathbf{Q}_{\mathrm{c}}(0) \\
& =\left[\begin{array}{cccccc}
\mathbf{A}_{\mathbf{1}} & \mathbf{A}_{2} & \cdots & \mathbf{A}_{\mathbf{M}_{\mathrm{s}}} & \cdots & \mathbf{A}_{\mathbf{K}-\mathbf{k}_{\mathrm{th}}-\mathbf{1}} \\
\mathbf{A}_{\mathbf{0}} & \mathbf{A}_{\mathbf{1}} & \cdots & \mathbf{A}_{\mathbf{M}_{\mathrm{s}}-\mathbf{1}} & \cdots & \mathbf{A}_{\mathbf{K}-\mathbf{k}_{\mathrm{th}}} \\
\vdots & \vdots & \ddots & \vdots & \ddots & \vdots \\
\mathbf{A}_{-\mathbf{M}_{\mathrm{s}}} & \mathbf{A}_{-\left(\mathbf{M}_{\mathrm{s}}-1\right)} & \cdots & \mathbf{A}_{\mathbf{0}} & \cdots & \mathbf{A}_{\mathbf{K}-\mathbf{k}_{\mathrm{th}}-\mathbf{M}_{\mathrm{s}}-\mathbf{1}} \\
\mathbf{0} & \mathbf{A}_{-\mathbf{M}_{\mathrm{s}}} & \cdots & \mathbf{A}_{-1} & \cdots & \mathbf{A}_{\mathbf{K}-\mathbf{k}_{\mathrm{th}}-\mathbf{M}_{\mathrm{s}}-\mathbf{2}} \\
\vdots & \vdots & \ddots & \vdots & \ddots & \vdots \\
\mathbf{0} & \mathbf{0} & \cdots & \mathbf{A}_{-\mathbf{M}_{\mathrm{s}}} & \cdots & \mathbf{A}_{\mathbf{0}}
\end{array}\right], \\
& \mathbf{Q}_{\mathbf{c}}(\ell)=\left[\begin{array}{ccccc}
\mathbf{0} & \mathbf{0} & \cdots & \mathbf{0} & \mathbf{A}_{\mathrm{K}-\mathbf{k}_{\mathrm{th}}-\mathbf{1}+\ell} \\
\mathbf{0} & \mathbf{0} & \cdots & \mathbf{0} & \mathbf{A}_{\mathrm{K}-\mathbf{k}_{\mathrm{th}}+\ell} \\
\vdots & \vdots & \ddots & \vdots & \vdots \\
\mathbf{0} & \mathbf{0} & \cdots & \mathbf{0} & \mathbf{A}_{\mathrm{K}-\mathbf{k}_{\mathrm{th}}-\mathbf{M}_{\mathrm{s}}-\mathbf{1}+\ell} \\
\mathbf{0} & \mathbf{0} & \cdots & \mathbf{0} & \mathbf{A}_{\mathrm{K}-\mathbf{k}_{\mathrm{th}}-\mathbf{M}_{\mathrm{s}}-2+\ell} \\
\vdots & \vdots & \ddots & \vdots & \vdots \\
\mathbf{0} & \mathbf{0} & \cdots & \mathbf{0} & \mathbf{A}_{\ell}
\end{array}\right], \quad \forall \ell \geq 1 .
\end{aligned}
$$

The matrix $\mathbf{Q}_{\mathbf{c}}(0)$ consists of the probabilities of events which make state transitions within $\Omega_{c}$ without any packet loss; however, the matrix $\mathbf{Q}_{\mathbf{c}}(\ell), \ell \geq 1$, consists of the probabilities of events which make state transitions within $\Omega_{c}$ with the loss of $\ell$ packets. Similarly, the submatrix $\mathbf{Q}_{\mathbf{n c}, \mathbf{c}}$ in (9) can also be considered as

$$
\mathbf{Q}_{\mathbf{n c}, \mathbf{c}}=\mathbf{Q}_{\mathbf{n c}, \mathbf{c}}(0)+\sum_{\ell=1}^{\infty} \mathbf{Q}_{\mathbf{n c}, \mathbf{c}}(\ell),
$$

where

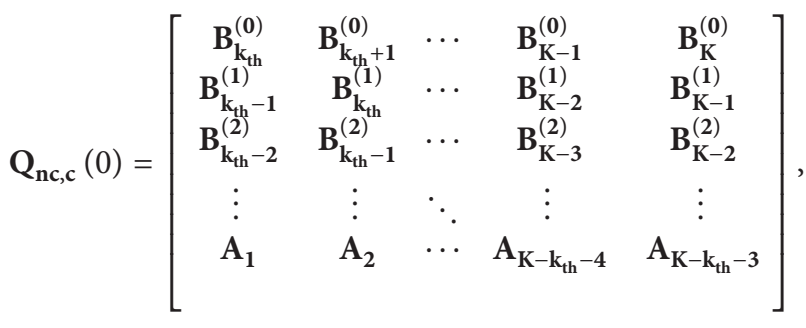

$$
\begin{aligned}
& \mathbf{Q}_{\mathbf{n c}, \mathbf{c}}(\ell)=\left[\begin{array}{ccccc}
\mathbf{0} & \mathbf{0} & \cdots & \mathbf{0} & \mathbf{B}_{\mathrm{K}+\ell}^{(\mathbf{0})} \\
\mathbf{0} & \mathbf{0} & \cdots & \mathbf{0} & \mathbf{B}_{\mathrm{K}-\mathbf{1}+\ell}^{(1)} \\
\mathbf{0} & \mathbf{0} & \cdots & \mathbf{0} & \mathbf{B}_{\mathrm{K}-2+\ell}^{(2)} \\
\vdots & \vdots & \ddots & \vdots & \vdots \\
\mathbf{0} & \mathbf{0} & \cdots & \mathbf{0} & \mathbf{A}_{\mathrm{K}-\mathbf{k}_{\mathrm{th}}-3+\ell}
\end{array}\right], \quad \forall \ell \geq 1 .
\end{aligned}
$$

The matrix $\mathbf{Q}_{\mathbf{n c}, \mathbf{c}}(0)$ consists of the probabilities of events which make a transition from a state in $\Omega_{n c}$ to a state in $\Omega_{c}$ without any packet loss; and the matrix $\mathbf{Q}_{\mathbf{n c}, \mathbf{c}}(\ell), \ell \geq$ 1 , consists of the probabilities of events which make such transition with the loss of $\ell$ packets.

Note that the behavior of the queueing system during a congested period can be described by the transient Markov chain. For a state $(k, j, i)$ in $\Omega_{c}$, let $p_{(k, j, i), c}(n, \ell)$ be the probability that the state of the transient Markov chain enters $(k, j, i)$ with a total of $\ell$ packets lost after $n$ transitions. Let $\mathbf{p}_{\mathbf{c}}(n, \ell)$ be an $\left|\Omega_{c}\right|$-vector whose $(k, j, i)$ th entry is $p_{(k, j, i), c}(n, \ell)$. The initial vector $\mathbf{p}_{\mathbf{c}}(0, \ell)$ can be determined by the behavior of the queueing system during a previous noncongested period as

$$
\begin{aligned}
\mathbf{p}_{\mathbf{c}}(0, \ell) & =\left(\sum_{n=1}^{\infty} \boldsymbol{\beta}_{\mathbf{n c}} \mathbf{Q}_{\mathbf{n c}}{ }^{n-1}\right) \mathbf{Q}_{\mathbf{n c}, \mathbf{c}}(\ell) \\
& =\boldsymbol{\beta}_{\mathbf{n c}}\left(\mathbf{I}-\mathbf{Q}_{\mathbf{n c}}\right)^{-1} \mathbf{Q}_{\mathbf{n c}, \mathbf{c}}(\ell), \quad \forall \ell \geq 0 .
\end{aligned}
$$

In addition, the vectors $\mathbf{p}_{\mathbf{c}}(n, \ell), n \geq 1$, and $\ell \geq 0$ can be obtained recursively by

$$
\mathbf{p}_{\mathbf{c}}(n, \ell)=\sum_{i=0}^{\ell} \mathbf{p}_{\mathbf{c}}(n-1, \ell-i) \mathbf{Q}_{\mathbf{c}}(i) \text {. }
$$

Let $p_{\text {loss, }}(n, \ell)$ be the probability that there is a total of $\ell$ packets lost during a congested period of length $n$. Then,

$$
p_{\text {loss }, c}(n, \ell)=\mathbf{p}_{\mathbf{c}}(n-1, \ell) \mathbf{Q}_{\mathbf{c}, \mathbf{n c}} \mathbf{e}, \quad \forall n \geq 1, \ell \geq 0 .
$$


The generating function $\widehat{p}_{\text {loss }, c}\left(z_{1}, z_{2}\right)$ of $p_{\text {loss }, c}(n, \ell)$, that is, $\widehat{p}_{\text {loss }, c}\left(z_{1}, z_{2}\right)=\sum_{n=1}^{\infty} \sum_{\ell=0}^{\infty} p_{\text {loss }, c}(n, \ell) z_{1}^{n} z_{2}^{\ell}$, is

$$
\begin{aligned}
\widehat{p}_{\text {loss }, \mathrm{c}}\left(z_{1}, z_{2}\right)= & z_{1} \widehat{\mathbf{p}}_{\mathbf{c}}\left(z_{1}, z_{2}\right) \mathbf{Q}_{\mathbf{c}, \mathbf{n c}} \mathbf{e} \\
= & z_{1} \boldsymbol{\beta}_{\mathbf{n c}}\left(\mathbf{I}-\mathbf{Q}_{\mathbf{n c}}\right)^{-1} \\
& \times \widehat{\mathbf{Q}}_{\mathbf{n c}, \mathbf{c}}\left(z_{2}\right)\left(\mathbf{I}-z_{1} \widehat{\mathbf{Q}}_{\mathbf{c}}\left(z_{2}\right)\right)^{-1} \mathbf{Q}_{\mathbf{c}, \mathbf{n c}} \mathbf{e}
\end{aligned}
$$

where $\widehat{\mathbf{Q}}_{\mathbf{n c , c}}\left(z_{2}\right)=\sum_{\ell=0}^{\infty} \mathbf{Q}_{\mathbf{n c}, \mathbf{c}}(\ell) z_{2}^{\ell}$ and $\widehat{\mathbf{Q}}_{\mathbf{c}}\left(z_{2}\right)=$ $\sum_{\ell=0}^{\infty} \mathbf{Q}_{\mathbf{c}}(\ell) z_{2}^{\ell}$. Now, the average total number of packets lost during a congested period, denoted as $E\left[X_{\text {loss }, c}\right]$, can be calculated as

$$
\begin{aligned}
E\left[X_{\text {loss }, c}\right]= & \left.\frac{\partial}{\partial z_{2}} \widehat{\mathbf{p}}_{\text {loss }, \mathbf{c}}\left(z_{1}, z_{2}\right)\right|_{z_{1}=1, z_{2}=1} \\
= & \boldsymbol{\beta}_{\mathbf{n c}}\left(\mathbf{I}-\mathbf{Q}_{\mathbf{n c}}\right)^{-1}\left(\sum_{\ell=1}^{\infty} \ell \mathbf{Q}_{\mathbf{n c}, \mathbf{c}}(\ell)\right) \mathbf{e} \\
& +\boldsymbol{\beta}_{\mathbf{c}}\left(\mathbf{I}-\mathbf{Q}_{\mathbf{c}}\right)^{-1}\left(\sum_{\ell=1}^{\infty} \ell \mathbf{Q}_{\mathbf{c}}(\ell)\right) \mathbf{e}
\end{aligned}
$$

by (22). Consequently, the packet loss probability $P_{\text {loss }, c}$ during a congested period can be obtained by

$$
P_{\mathrm{loss}, c}=\frac{E\left[X_{\mathrm{loss}, c}\right]}{\lambda E\left[L_{c}\right]}
$$

where $E\left[L_{c}\right]$ is the average length of a congested period in (14) and $\lambda$ is the fundamental arrival rate of the arrival traffic.

3.2. Steady State Performance Measure. Let $\mathbf{x}=$ $\left[\mathbf{x}_{0}, \mathbf{x}_{1}, \ldots, \mathbf{x}_{K}\right]$, with $\mathbf{x}_{k}=\left[x_{k, 1,1}, x_{k, 1,2}, \ldots, x_{k, m_{a}, m_{s}}\right] \forall k$, be the steady state probability vector of the queueing system; that is,

$$
\begin{aligned}
& \mathbf{x Q}=\mathbf{x}, \\
& \mathbf{x e}=1 .
\end{aligned}
$$

It follows from (25) that

$$
\mathbf{x}(\mathbf{I}-\mathbf{Q})=\mathbf{0} .
$$

Notice that the system of linear equations (26) and (27) has finite dimension; thus, some algorithms given in the literature can be used to solve the system of linear equations, such as Gauss elimination method and Gauss-Seidel method. The Gauss-Seidel method is usually more efficient and more effective than the Gauss elimination method when the size of the transition probability matrix is large. The number of iterations required to achieve the desired convergence is not usually known in advance and the resulting distributions have round-off errors associated with them. For more details on above algorithms, see Alfa [18].

Let $X_{\text {loss }}$ denote the number of packets lost during a time slot, with only long term packet loss probability considered as significant. Now, the expected value $E\left[X_{\text {loss }}\right]$ of $X_{\text {loss }}$ can be evaluated as

$$
\begin{aligned}
E\left[X_{\text {loss }}\right]= & \mathbf{x}_{0}\left(\sum_{i=1}^{\infty} i \mathbf{D}_{\mathbf{K}+\mathbf{i}} \otimes \mathbf{H}\right) \mathbf{e} \\
& +\sum_{k=1}^{M_{s}-1} \mathbf{x}_{k}\left(\sum_{i=1}^{\infty} i\left(\sum_{j=0}^{k} \mathbf{D}_{\mathbf{K}-\mathbf{k}+\mathbf{i}+\mathbf{j}} \otimes \mathbf{H}_{\mathbf{j}}\right)\right) \mathbf{e} \\
& +\sum_{k=M_{s}}^{K} \mathbf{x}_{k}\left(\sum_{i=1}^{\infty} i\left(\sum_{j=0}^{M_{s}} \mathbf{D}_{\mathbf{K}-\mathbf{k}+\mathbf{i}+\mathbf{j}} \otimes \mathbf{H}_{\mathbf{j}}\right)\right) \mathbf{e} .
\end{aligned}
$$

Consequently, the long term packet loss probability, denoted by $P_{\text {loss }}$, is

$$
P_{\text {loss }}=\frac{E\left[X_{\text {loss }}\right]}{\lambda},
$$

where $\lambda$ is the fundamental arrival rate of the packet stream as in (3).

\section{Application}

We consider an end-to-end wireless transmission system with adaptive modulation and coding (AMC) scheme in the physical layer to provide streaming media service. We experiment an arrival process described by a DBMAP which can capture time correlations commonly observed in VBR traffic such as MPEG coded streaming video. On the transmitter side, streaming media packets are buffered in a queue with finite length $K$, which operates in a first-in-first-out (FIFO) manner. On the receiver side, a selector determines the modulation mode and coding rate and sends them back to the transmitter through an error-free feedback channel. Due to different modulation modes, each packet may contain one or more streaming media packets.

In AMC scheme, the modulation mode and coding rate are chosen depending on the time-varying channel conditions. AMC is employed at the physical layer with $m_{s}$ transmission modes corresponding to different channel state. We assume that when the channel is in state $k$, the transmitter transmits $c_{k}$ packets. We further assume that $c_{1}=0$. For convenience, let $c_{m_{s}}=M_{s}$.

For flat channels, we adopt the general Nakagami- $m$ model to describe the received signal-to-noise ratio (SNR) A statistically, which is a random variable with Gamma probability density function

$$
f_{A}(x)=\frac{m^{m} x^{m-1}}{\xi^{m} \Gamma(m)} \exp \left(-\frac{m x}{\xi}\right),
$$

where $\xi$ is the expected value of $A, \Gamma(m)=\int_{0}^{\infty} t^{m} \exp (-t) d t$, and $m$ is the Nakagami- $m$ fading parameter. We partition the entire SNR range into $m_{s}+1$ nonoverlapping consecutive intervals with boundaries denoted as $0=A_{0}<A_{1}<\cdots<$ $A_{m_{s}-1}<A_{m_{s}}=\infty$. The algorithm for determining the boundaries with the prescribed packet error rate is described in detail in [19]. Specifically, mode $n$ is chosen when the 
received SNR is in the interval $\left[A_{n-1}, A_{n}\right)$. To capture the variations of the multistate Nakagami fading channel, we employ the finite state Markov channel model used in [19]. For a packet transmission system, we assume that a onestep transition in the Markov channel model corresponds to channel state transition after one packet time period $T_{p}$. Then, the Nakagami- $m$ fading is said to be in state $n, n=$ $1,2, \ldots, m_{s}$, if the received SNR is in the interval $\left[A_{n-1}, A_{n}\right)$. We allow transitions from a given state to its two adjacent states only. Let $N_{n}, n \in\left\{1, \ldots, m_{s}\right\}$, be the expected number of times per second the received SNR passes downward across the threshold $A_{n}$. We have

$$
\begin{array}{r}
N_{n}=\sqrt{\frac{2 \pi m A_{n}}{\xi}} \frac{f_{d}}{\Gamma(m)}\left(\frac{m A_{n}}{\xi}\right)^{m-1} \exp \left(-\frac{m A_{n}}{\xi}\right), \\
n=1, \ldots, m_{s},
\end{array}
$$

where $f_{d}$ is the mobility-induced Doppler spread. Based on the slow fading assumption of the Rayleigh fading channel, the level crossing rate should be much smaller than the value of the packet transmission rate $1 / T_{p}$. Thus, we can get the approximated value of transition probabilities as follows:

$$
\begin{gathered}
h_{n, n+1} \approx \frac{N_{n+1} T_{p}}{P_{r}(n)}, \quad n \in\left\{1,2, \ldots, m_{s}-1\right\}, \\
h_{n, n-1} \approx \frac{N_{n} T_{p}}{P_{r}(n)}, \quad n \in\left\{2, \ldots, m_{s}\right\}, \\
h_{n, n}=1-h_{n, n-1}-h_{n, n+1}, \quad n \in\left\{2, \ldots, m_{s}-1\right\}, \\
h_{1,1}=1-h_{1,2}, \\
h_{m_{s}, m_{s}}=1-h_{m_{s}, m_{s}-1} .
\end{gathered}
$$

The transition matrix $H$ of FSMC is

$$
\mathbf{H}=\left(\begin{array}{cccccc}
h_{1,1} & h_{1,2} & 0 & 0 & \cdots & 0 \\
h_{2,1} & h_{2,2} & h_{2,3} & 0 & \ldots & 0 \\
0 & h_{3,2} & h_{3,3} & h_{3,4} & \ldots & 0 \\
\vdots & \vdots & \vdots & \vdots & \vdots & \vdots \\
0 & 0 & \cdots & h_{m_{s}-1, m_{s}-2} & h_{m_{s}-1, m_{s}-1} & h_{m_{s}-1, m_{s}} \\
0 & 0 & \cdots & 0 & h_{m_{s}, m_{s}-1} & h_{m_{s}, m_{s}}
\end{array}\right) .
$$

Finally, consider a Nakagami-m fading channel which can be modeled by a DBMSP which is defined by matrices $\mathbf{H}_{\mathbf{k}}, k=0, \ldots, M_{s}$, whose elements $h_{i j}(k)=\operatorname{Pr}(k, j \mid i)$ are the conditional probabilities of transferring from channel state $i$ to channel state $j$ and producing $k$ packets successfully transmitted. Then, it can be found as follows:

$$
\mathbf{H}_{\mathbf{c}_{\mathbf{k}}}=\operatorname{Diag}\left(\mathbf{e}_{\mathbf{k}}\right) \mathbf{H} \text {, }
$$

where $\mathbf{e}_{\mathbf{k}}$ is the vector which has all zero entries except in the $k$ th entry with 1 .

\section{Numerical Results}

In this section, we present some numerical results obtained on the basis of an FSMC of packet transmission mode induced by the Rayleigh fading $[19,20]$. We remark that this is just an example to illustrate the application of our analysis. Discussion about appropriate ways to model fading channels is not the focus here. We refer to the vast literature on the topic for more detail. Consider the case $F=12 \mathrm{~dB}$ and let $f_{D} T_{p}=$ 0.02 be the value of the Doppler frequency normalized to the packet transmission rate, which determines the process memory span. The fading process is approximated by the FSMC with the following matrix probability:

$$
\mathbf{H}=\left(\begin{array}{cccccc}
0.649 & 0.351 & 0 & 0 & 0 & 0 \\
0.0224 & 0.909 & 0.0686 & 0 & 0 & 0 \\
0 & 0.073 & 0.8687 & 0.061 & 0 & 0 \\
0 & 0 & 0.1521 & 0.7273 & 0.1206 & 0 \\
0 & 0 & 0 & 0.1315 & 0.7823 & 0.0862 \\
0 & 0 & 0 & 0 & 0.0709 & 0.9291
\end{array}\right)
$$

The service rate per state of wireless link with AMC is

$$
\Lambda_{\mathrm{s}}=\left(\begin{array}{llllll}
0 & 1 & 2 & 3 & 6 & 9
\end{array}\right) \text { packets/time slot. }
$$

We can find $\mathbf{H}_{\mathbf{k}}, k=0, \ldots, 9$, by (34).

The time is slotted such that the unit time is equal to the packet transmission time, which is equal to $2 \mathrm{~ms}$. The numerical results are computed by the algorithm developed in Section 3.

In [21], Skelly et al. proposed an eight-state MMPP to model a compressed version of the Star Wars movie. In [4], Heyman and Lucantoni provided evidence that a DMMPP is a good model for internet traffic at the packet/byte level. In [5], Salvador et al. proposed a parameter fitting procedure using superposed two-state MMPP that leads to accurate estimates of queueing behavior for network traffic exhibiting long-range-dependent behavior. Based on the above observation, we experiment an arrival process described by a DMMPP which can capture time correlations commonly observed in VBR traffic such as MPEG coded streaming video. In DMMPP, packets arrive according to a Poisson process whose instantaneous rate is a function of the state of a discrete-time finite Markov chain. Thus, an DMMPP can be represented by a pair of matrices $\left(D, \Lambda_{a}\right)$, with the first matrix being the transition matrix of the Markov chain and the second being a diagonal matrix specifying the arrival intensity associated with each state of the Markov chain. Let the rate matrix $\Lambda_{\mathbf{a}}=\left(\lambda_{1}, \ldots, \lambda_{m_{a}}\right)$. This definition of a DMMPP coincides with a DBMAP with representation $\left\{\mathbf{D}_{\mathbf{i}}\right\}$, where

$$
\mathbf{D}_{\mathbf{i}}=\operatorname{Diag}\left(\frac{e^{-\lambda_{1}}\left(\lambda_{1}\right)^{i}}{i !}, \ldots, \frac{e^{-\lambda_{n}}\left(\lambda_{n}\right)^{i}}{i !}\right) \mathbf{D}
$$




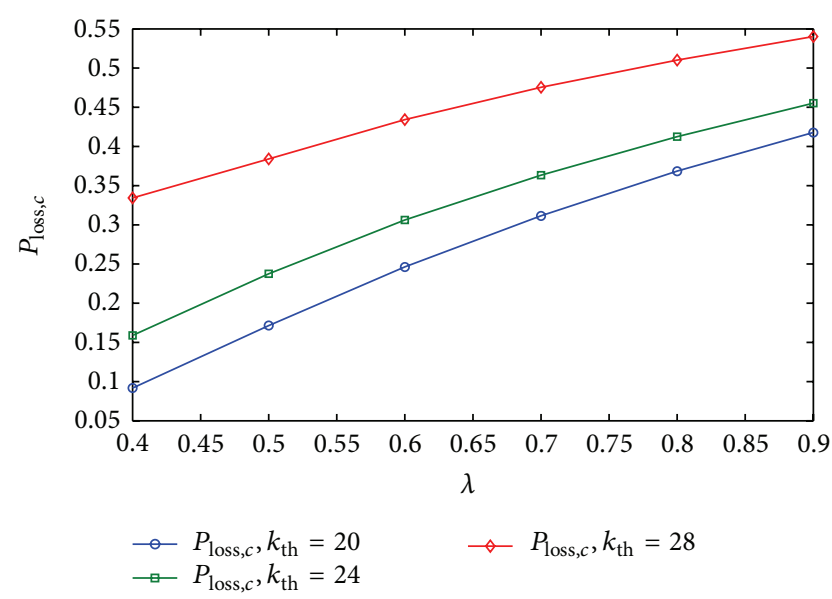

FIGURE 1: Short term packet loss probability with buffer capacity $K=$ 30.

The irreducible stochastic matrix $\mathbf{D}=\sum_{i=0}^{\infty} \mathbf{D}_{\mathbf{k}}$. In our experimental studies, the numerical values for the parameters used are

$$
\begin{gathered}
\mathbf{D}=\left(\begin{array}{ccccc}
0.35 & 0.3 & 0.2 & 0.1 & 0.05 \\
0.2 & 0.3 & 0.2 & 0.18 & 0.12 \\
0.14 & 0.22 & 0.3 & 0.21 & 0.13 \\
0.14 & 0.21 & 0.22 & 0.28 & 0.15 \\
0.12 & 0.23 & 0.25 & 0.22 & 0.18
\end{array}\right) \\
\Lambda_{\mathrm{a}}=\left(\begin{array}{lllll}
0.6 & 1.2 & 1.8 & 3.2 & 4.1
\end{array}\right)
\end{gathered}
$$

We also multiply the arrival rates $\Lambda_{a}$ by a factor $w=1.3535,1.2018,1.0502,0.8999,0.7499,0.5997$, so that the queue will have different load conditions. The buffer capacity $K$ is taken to be 30 .

Figure 1 illustrates the packet loss probability $P_{\text {loss }, c}$ during a congested period as a function of the traffic arrival rate and threshold setting. When the traffic arrival rate increases, it leads to a small increase in the short term loss probability of the arriving traffic. With the threshold setting $k_{t h}=20, P_{\text {loss }, c}$ increases from 0.09 to 0.4 with a traffic arrival rate $\lambda$ from 0.4 to 0.9 . With a traffic arrival rate $\lambda=0.6, P_{\text {loss }, c}$ increases from 0.25 to 0.43 with a threshold setting $k_{t h}$ from 20 to 28 . Due to the buffer limitations, $P_{\mathrm{drop}, c}$ is high under any load conditions. This finding confirms our postulation that the short term packet loss probability during a congested period is a significant performance measure of a queue.

Figure 2 summarizes the numerical results of the average lengths of a congested period $E\left[L_{c}\right]$ and a noncongested period $E\left[L_{n c}\right]$ with respect to the traffic arrival rate and threshold setting. As it is anticipated, $E\left[L_{c}\right]$ slightly varies with the load condition. Additionally, $E\left[L_{n c}\right]$ is significantly longer than that of a congested period under a light-tomoderate load condition; in addition, $E\left[L_{n c}\right]$ decreases with an increasing traffic arrival rate. According to Figure 2, $E\left[L_{c}\right]$ increases with an increasing traffic arrival rate. A cycle consists of a congested period and a noncongested period; decreasing the average length of the noncongested period by a larger factor would reduce the length of the cycle. This

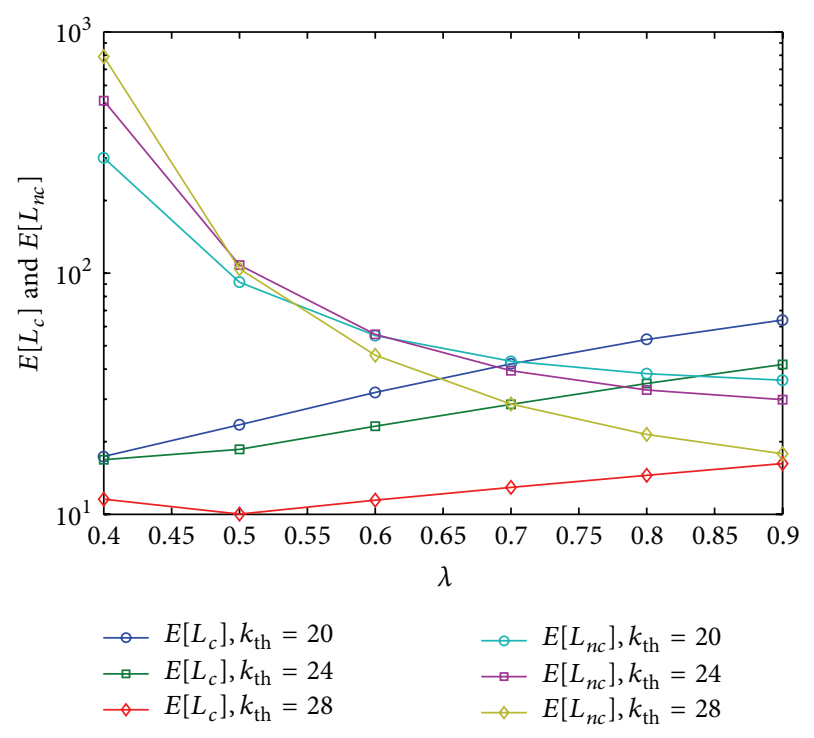

Figure 2: Average length of noncongested period and congested period with buffer capacity $K=30$.

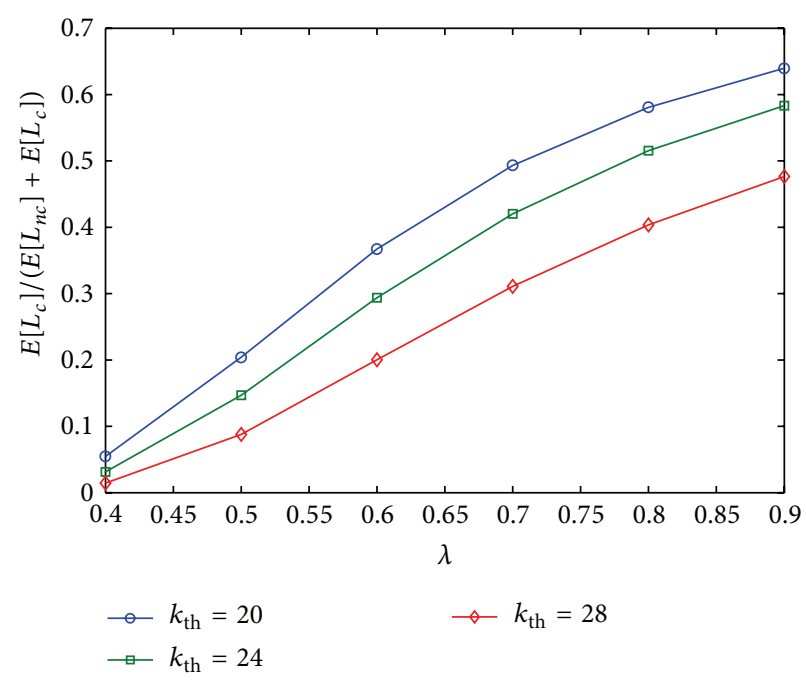

FIGURE 3: Probability that queuing system stays in congested period with buffer capacity $K=30$.

effect causes the system to frequently alternate congested periods and noncongested periods. Because the system alternates between noncongested periods and congested periods, increasing the traffic arrival rate $\lambda$ increases the probability $E\left[L_{c}\right] /\left(E\left[L_{c}\right]+E\left[L_{n c}\right]\right)$ that the system remains in the congested period. Increasing the system bandwidth reduces the average length of the congested period and increases the average length of the noncongested period.

According to Figure 3, $E\left[L_{c}\right] /\left(E\left[L_{c}\right]+E\left[L_{n c}\right]\right)$ increases with an increasing traffic arrival rate. However, $P_{\text {loss }, c}, E\left[L_{c}\right]$ and $E\left[L_{c}\right] /\left(E\left[L_{c}\right]+E\left[L_{n c}\right]\right)$ profoundly impact wireless communication based multimedia services. For instance, with traffic arrival rate $\lambda=0.7$ and threshold setting $k_{t h}=20$, $P_{\text {loss }, c}=0.31$ and $E\left[L_{c}\right]=42$ time slots, indicating that 


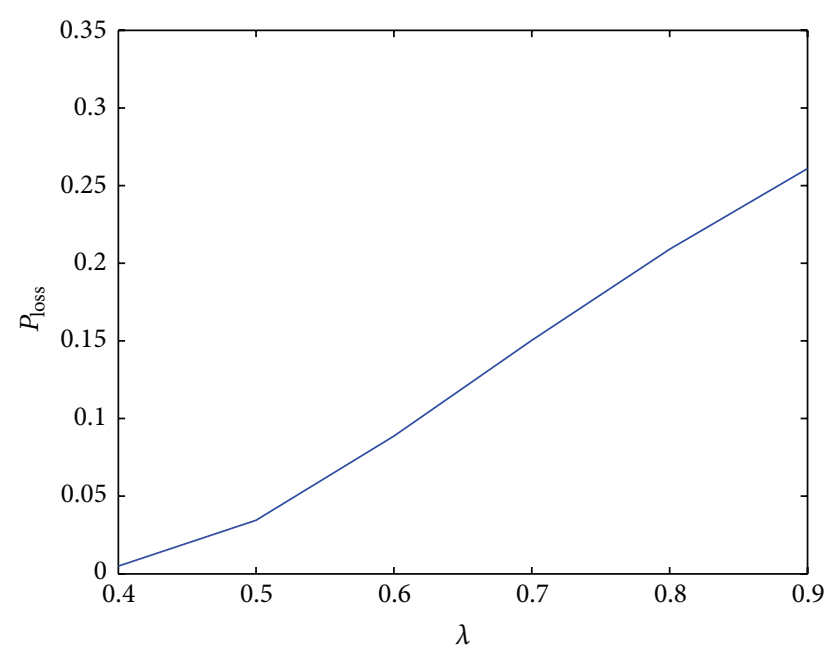

FIGURE 4: Long term packet loss probability with buffer capacity $K=$ 30 .

the packet loss probability during a congested period and the average length of a congested period are too high to provide multimedia services. According to Figure $4, P_{\text {loss }}$ increases with an increasing traffic arrival rate. Sensitivity of $P_{\text {loss }}$ to the traffic load is largely owing to $E\left[L_{c}\right] /\left(E\left[L_{c}\right]+E\left[L_{n c}\right]\right)$.

\section{Conclusions}

This paper applies matrix-analytic approach to investigate the loss behavior of Markovian-modulated batch-service queueing model with DBMAP. We have examined the congestion nature of packet loss by means of conditional statistics with respect to alternating congestion and non-congestion periods and evaluated the long term packet loss probabilities. By the conditional statistics, all of the four related performance measures are derived, including (1) a long term loss probability $P_{\text {loss }}$ and (2) the three short term measures, such as the average lengths $E\left[L_{c}\right]$ and $E\left[L_{n c}\right]$ of a congestion period and a non-congestion period, as well as conditional packet loss probability $P_{\text {loss }, c}$ during a congestion period. We use this queuing model to quantify the effects of multimedia services over a Rayleigh fading channel.

\section{Conflict of Interests}

The authors declare that there is no conflict of interests regarding the publication of this paper.

\section{Acknowledgment}

This work was supported by the National Science Council, Taiwan, under Grant NSC92-2213-E-027-047.

\section{References}

[1] M. F. Neuts, "Models based on the Markovian arrival process," IEICE Transactions on Communications, vol. 75, no. 12, pp. 1255$1265,1992$.
[2] C. Blondia and O. Casals, "Statistical multiplexing of VBR sources-a matrix-analytic approach," Performance Evaluation, vol. 16, no. 1-3, pp. 5-20, 1992.

[3] S. H. Kang, Y. H. Kim, D. K. Sung, and B. D. Choi, "An application of Markovian arrival process (MAP) to modeling superposed ATM cell streams," IEEE Transactions on Communications, vol. 50, no. 4, pp. 633-642, 2002.

[4] D. P. Heyman and D. Lucantoni, "Modeling multiple IP traffic streams with rate limits," IEEE/ACM Transactions on Networking, vol. 11, no. 6, pp. 948-958, 2003.

[5] P. Salvador, R. Valadas, and A. Pacheco, "Multiscale fitting procedure using Markov modulated Poisson processes," Telecommunication Systems, vol. 23, no. 1-2, pp. 123-148, 2003.

[6] Y.-C. Wang, C.-W. Liu, and C.-C. Lu, "Loss behavior in space priority queue with batch Markovian arrival process-discretetime case," Performance Evaluation, vol. 41, no. 4, pp. 269-293, 2000.

[7] P. P. Bocharov, Ch. D’Apiche, A. V. Pechinkin, and S. Salerno, "Stationary characteristics of the queueing system $G / M S P / 1 / r$," Autom Remote Control, no. 2, pp. 127-142, 2003.

[8] A. D. Banik and U. C. Gupta, "Analyzing the finite buffer batch arrival queue under Markovian service process: GI $I^{X} / M S P / 1 / N$," TOP, vol. 15, no. 1, pp. 146-160, 2007.

[9] U. C. Gupta and A. D. Banik, "Complete analysis of finite and infinite buffer GI/MSP/1 queue-a computational approach," Operations Research Letters, vol. 35, no. 2, pp. 273-280, 2007.

[10] Y.-C. Wang, J.-H. Chou, and S.-Y. Wang, "Loss pattern of $D B M A P / D M S P / 1 / K$ queue and its application in wireless local communications," Applied Mathematical Modelling, vol. 35, no. 4, pp. 1782-1797, 2011.

[11] S. K. Samanta and Z. G. Zhang, "Stationary analysis of a discrete-time GI/D-MSP/1 queue with multiple vacations," Applied Mathematical Modelling, vol. 36, no. 12, pp. 5964-5975, 2012.

[12] M. L. Chaudhry and U. C. Gupta, "Analysis of a finite-buffer bulk-service queue with discrete-Markovian arrival process: $D$ $M A P / G^{a, b} / 1 / N$," Naval Research Logistics, vol. 50, no. 4, pp. 345-363, 2003.

[13] A. D. Banik, "Queueing analysis and optimal control of $B M A P / G^{(a, b)} / 1 / N$ and BMAP/MSP $P^{(a, b)} / 1 / N$ systems," Computers and Industrial Engineering, vol. 57, no. 3, pp. 748-761, 2009.

[14] D. Claeys, K. Laevens, J. Walraevens, and H. Bruneel, "Complete characterisation of the customer delay in a queueing system with batch arrivals and batch service," Mathematical Methods of Operations Research, vol. 72, no. 1, pp. 1-23, 2010.

[15] D. Claeys, J. Walraevens, K. Laevens, and H. Bruneel, "Analysis of threshold-based batch-service queueing systems with batch arrivals and general service times," Performance Evaluation, vol. 68, no. 6, pp. 528-549, 2011.

[16] D. Claeys, B. Steyaert, J. Walraevens, K. Laevens, and H. Bruneel, "Analysis of a versatile batch-service queueing model with correlation in the arrival process," Performance Evaluation, vol. 70, no. 4, pp. 300-316, 2013.

[17] A. Graham, Kronecker Products and Matrix Calculus: With Applications, Ellis Horwood Series in Mathematics and its Applications, Ellis Horwood, Chichester, UK, 1981.

[18] A. S. Alfa, Queueing Theory for Telecommunicaions, Springer, New York, NY, USA, 2010.

[19] Q. Liu, S. Zhou, and G. B. Giannakis, "Queuing with adaptive modulation and coding over wireless links: cross-layer analysis 
and design," IEEE Transactions on Wireless Communications, vol. 4, no. 3, pp. 1142-1153, 2005.

[20] S. Vassilaras, "A cross-layer optimized adaptive modulation and coding scheme for transmission of streaming media over wireless links," Wireless Networks, vol. 16, no. 4, pp. 903-914, 2010.

[21] P. Skelly, M. Schwartz, and S. Dixit, "Histogram-based model for video traffic behavior in an ATM multiplexer," IEEE/ACM Transactions on Networking, vol. 1, no. 4, pp. 446-458, 1993. 


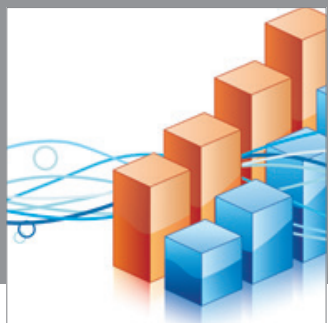

Advances in

Operations Research

mansans

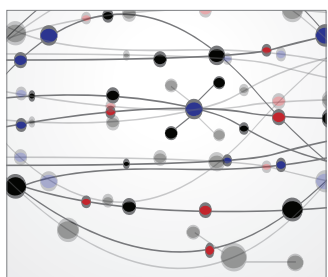

The Scientific World Journal
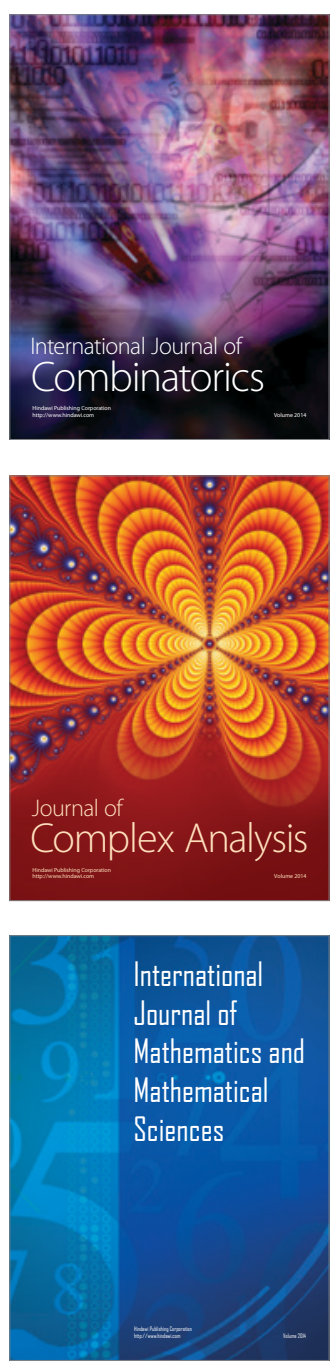
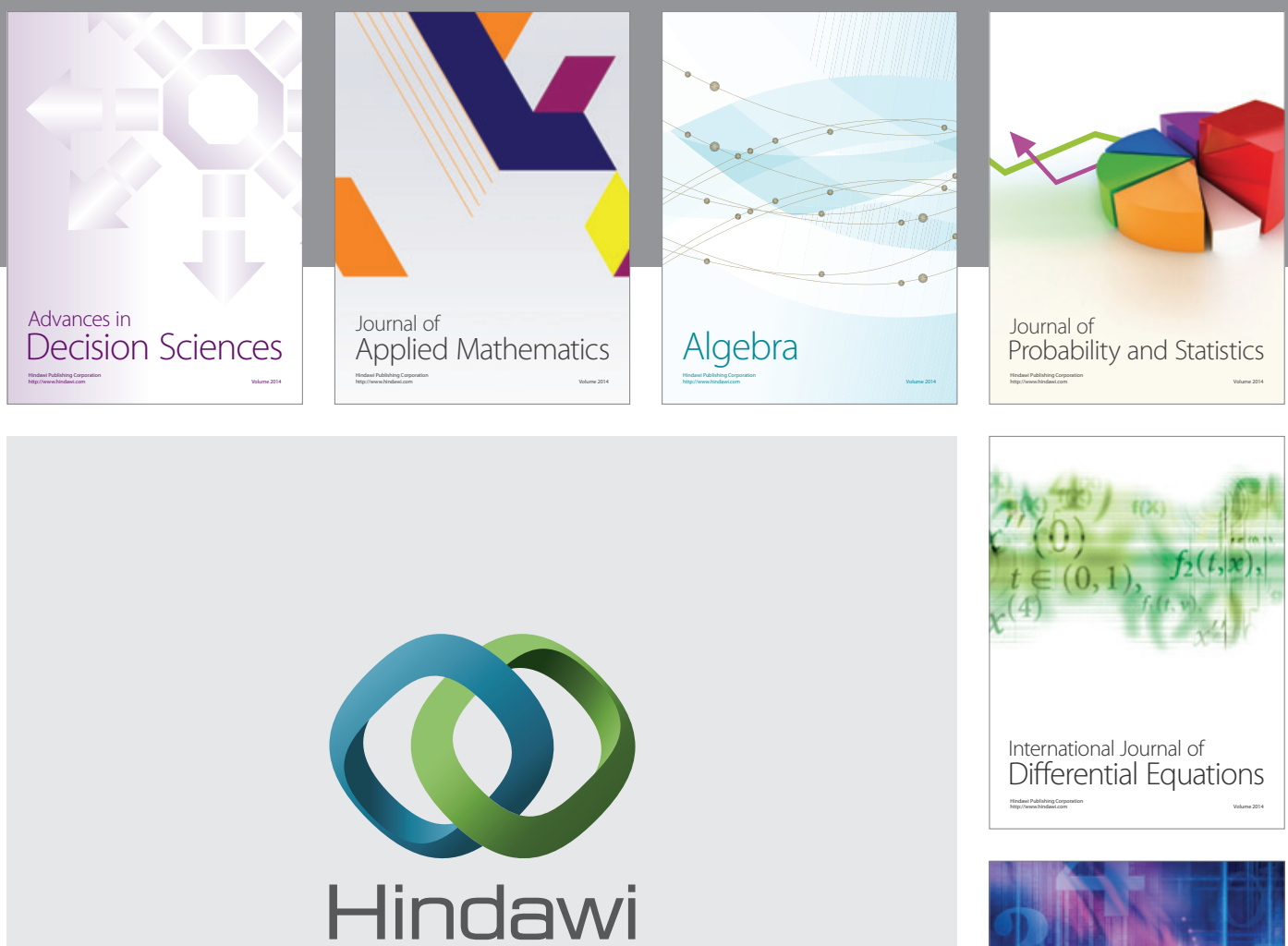

Submit your manuscripts at http://www.hindawi.com
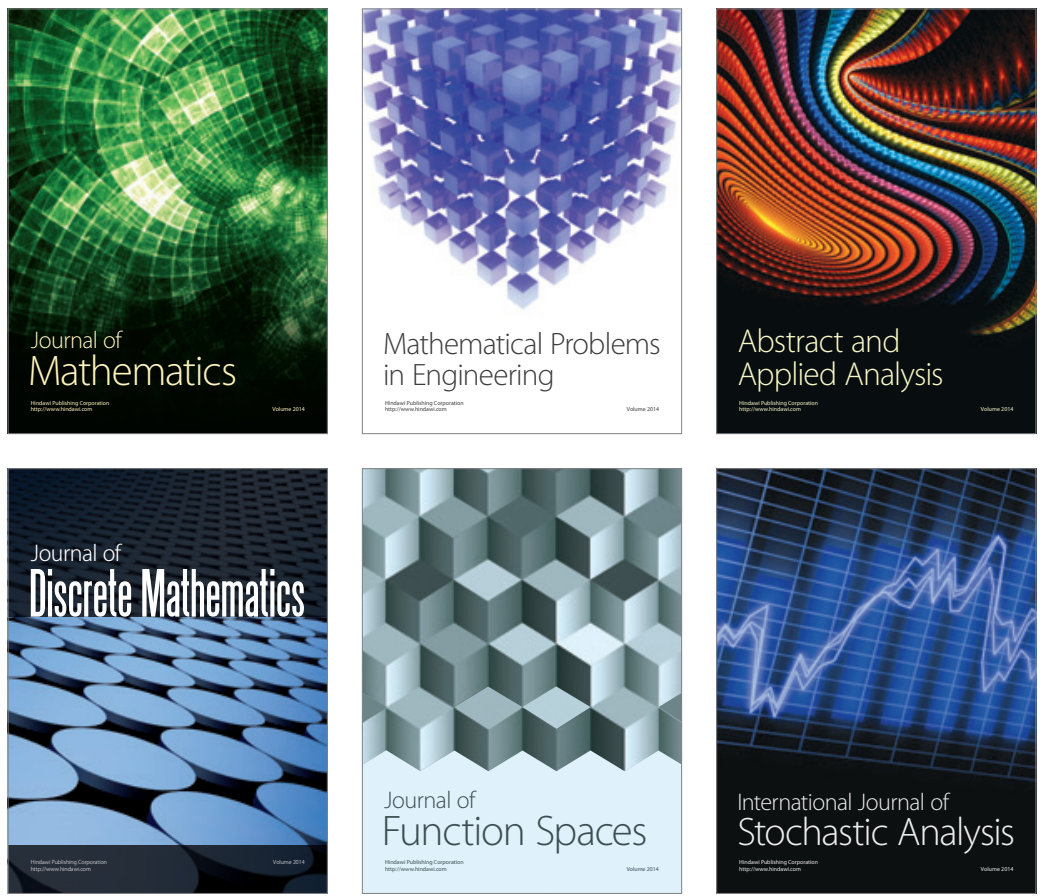

Journal of

Function Spaces

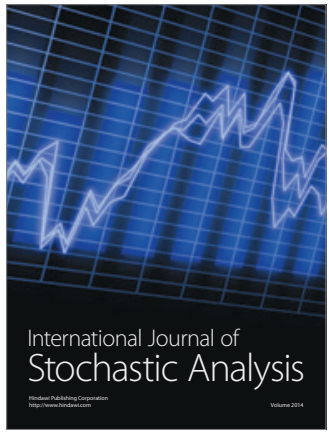

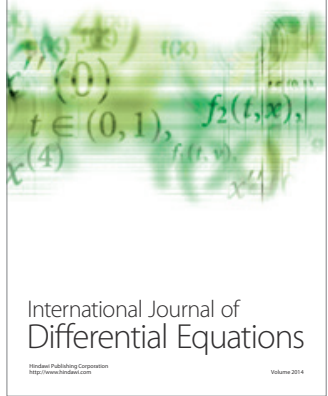
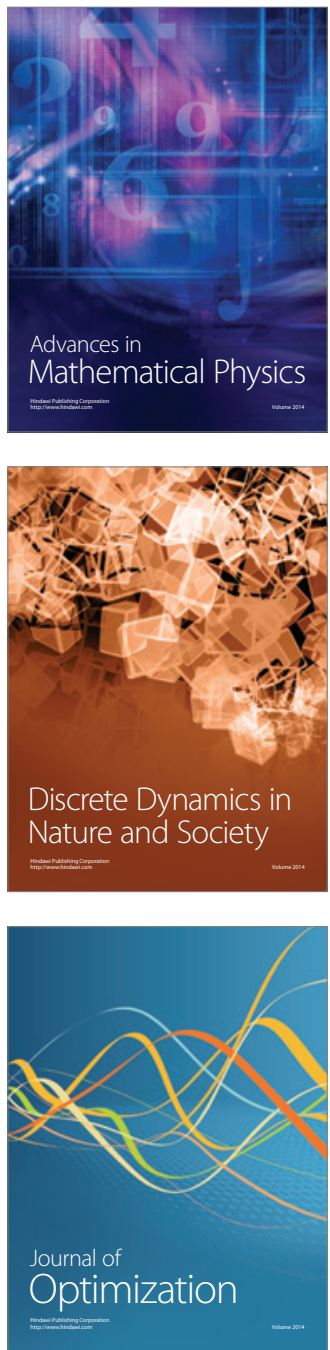